\title{
PARADIGMAS TECNOLÓGICOS E ESTÁGIOS DE DIVERSIFICAÇÃO TECNOLÓGICA
}

\author{
Vanessa de Lima Avanci ${ }^{*}$ \\ Ana Urraca Ruiz ${ }^{* *}$
}

recibido: abril 2015 - aceptado: junio 2015

\begin{abstract}
Resumo
Este artigo contrasta a existência de estágios de diversificação tecnológica para um grupo de países da Ásia e da América Latina a partir dos anos 80, quando novos paradigmas tecnológicos surgiram. Assumindo que mudanças na especialização tecnológica estão associadas às mudanças na especialização produtiva, este trabalho busca evidências sobre uma evolução em estágios das estruturas tecnológicas, ou seja, uma fase inicial correspondente a uma elevada especialização em poucos campos técnicos, seguida de uma fase de crescente diversificação da base tecnológica que acompanha a fase de diversificação produtiva e de desenvolvimento tecnológico dos paradigmas. No entanto, a evolução dos paradigmas está condicionada a trajetórias que ao longo do tempo levam à redução de oportunidades e, em decorrência, a uma reversão (concentração) das estruturas com um formato esperado de $U$ da tendência da evolução. $O$ trabalho mostra que, em geral, os países reduziram suas distâncias tecnológicas em relação a uma área ampla de referência diversificando suas estruturas tecnológicas. No entanto, foram identificados distintos padrões de diversificação/especialização que devem estar associados a outros fatores além do nível de desenvolvimento dos países, especialmente ao papel desempenhado por Grandes Corporações Transnacionais.
\end{abstract}

Código JEL: $\mathrm{O} 33$

Palavras-chave: Mudança tecnológica, especialização tecnológica, padrão de diversificação, estágios de diversificação.

* Doutoranda do Programa de Pós-Graduação de Economia da Universidade Federal Fluminense. E-mail: vanessa.avanci@gmail.com

** Professora Associada do Departamento de Economia da Universidade Federal Fluminense. E-mail: anaurracaruiz@gmail.com 


\begin{abstract}
This paper tests the existence of stages of technological diversification for a group of Asian and Latin-American countries since the late eighties; this is, when new technological paradigms emerged. Assuming that changes in technological specializations are linked to changes in productive specializations, this work look for evidences about the evolutions of technological structures in stages, this is, an initial phase of strong specialization in a few technical fields followed by a growing diversification, all along the period of expansion of techno-scientific paradigms. As the paradigms reduce their possibilities given the technological trajectories, technological opportunities become lower and $U$ format-curve is expected. In general terms, countries reduced their technological distances with a wide reference area making more diversified their technological profiles. Nevertheless, different patterns of diversification/specialization were identified which must obey to other factors beyond the catching-up process. Among these factors, the role of technological leaders and transnational corporations is quite relevant.
\end{abstract}

JEL Code: $\mathrm{O} 33$

Keywords: Technological change, technological specialization, diversification pattern, stages of diversification.

\title{
INTRODUÇÃO
}

A estrutura tecnológica de um país pode ser compreendida como a distribuição de sua atividade tecnológica entre uma diversidade de campos de conhecimento tecnológico, ou seja, a estrutura tecnológica reflete a composição das atividades de inovação entre campos de conhecimento tecnológico (Archibugi e Pianta, 1994). As mudanças registradas nas estruturas tecnológicas normalmente respondem à realocação de recursos (insumos) tecnológicos de umas tecnologias a outras, o que representa a dimensão tecnológica da mudança estrutural (UrracaRuiz, 2013a). Esta realocação da P\&D pode obedecer a três conjuntos diferentes de forças: autônomas, induzidas e estruturais (ibidem, 2013).

As forças de caráter estrutural fazem referência aos vínculos entre a estrutura tecnológica e produtiva, dado que o conhecimento tecnológico é construído sobre as competências produtivas das firmas com um elevado grau de especificidade setorial (Malerba e Montobbio, 2003). As atividades de inovação ocorrem nas atividades rotineiras da firma, sendo estas classificadas como produtivas, tecnológicas e organizacionais (Nelson e Winter, 1982; Teece, Rumelt, Dosi e Winter 
1994). O desenvolvimento de competências surge do aprendizado decorrente da resolução de problemas nessas três dimensões em atividades como P\&D formal e informal, relações com fornecedores e usuários e outros agentes externos à firma ou o estabelecimento de estratégias corporativas de crescimento interno (ibidem). Como a construção de competências tecnológicas está associada às competências produtivas e como os processos de inovação (insumos e resultados) são específicos de cada indústria, as estruturas tecnológicas são determinadas endogenamente. Desta forma, é plausível pensar que mudanças na estrutura tecnológica estão fortemente vinculadas a processos internos (nacionais) de mudança estrutural, especialmente quando estes decorrem de realocação de recursos produtivos após choques externos (por exemplo, a integração comercial). Nestes casos, as transformações na base de conhecimento técnico e científico nacional ocorrem em direção a uma composição tecnológica compatível com a nova base produtiva.

Associações semelhantes podem ser realizadas quando a mudança estrutural é observada como um processo natural do desenvolvimento. Imbs e Wackziarg (2003) apontaram que as estruturas produtivas se alteram ao longo do desenvolvimento econômico de acordo com diferentes estágios. Em estágios iniciais, as estruturas são fortemente concentradas em poucas indústrias (especializadas). Mas à medida que o desenvolvimento avança, as estruturas produtivas se diversificam. Em fases maduras do desenvolvimento, os países registrariam ainda uma volta à especialização. Assumindo esta evidência como um fato estilizado do desenvolvimento, este trabalho adota como hipótese que os movimentos das estruturas produtivas teriam um reflexo na evolução das estruturas tecnológicas. Ou seja, a realocação de fatores produtivos levaria a uma realocação de insumos tecnológicos estimulando a mobilidade tecnológica entre campos técnicos (Urraca-Ruiz, 2013b). Mais do que isso, a mobilidade tecnológica deveria seguir de alguma forma a mesma tendência 'especialização-diversificação-especialização' ao longo de um processo não só de desenvolvimento produtivo, mas também tecnológico. Porém, as possibilidades tecnológicas são muito maiores do que as possibilidades viáveis e socialmente aceitáveis que se tornam inovações (Perez, 2010), o que torna a base de conhecimentos técnicos e científicos mais ampla do que a base produtiva. Portanto, os estágios de diversificação tecnológica deverão ser mais extensos e menos acentuados que os da diversificação produtiva.

Sobre esta tendência geral, a evolução das estruturas tecnológicas pode ainda adquirir diferentes padrões de diversificação/especialização em função de cinco elementos: i) o aproveitamento nacional da direção e evolução de recentes paradigmas tecnológicos; ii) o padrão de inserção da $\mathrm{P} \& \mathrm{D}$ transnacional; iii) o tamanho do país; iv) a estabilidade dos líderes tecnológicos e v) a distância tecnológica do país com a 
fronteira tecnológica. Em primeiro lugar, os paradigmas tecno-científicos ampliam o escopo de possibilidades de inovação abrindo oportunidades em novos campos de conhecimento. A oportunidade tecnológica associada aos paradigmas tecnológicos estimula a realocação de recursos desde tecnologias com menor oportunidade para as de maior oportunidade. Paralelamente, os esforços de inovação em tecnologias relacionadas com novos paradigmas podem levar ao desenvolvimento de tecnologias similares ou afins, o que conduzirá a estruturas tecnológicas mais diversificadas. Uma vez que os paradigmas evoluem seguindo as trajetórias tecnológicas definidas pela seleção do mercado em forma de micro-paradigmas, o leque de oportunidades tecnológicas iniciais se reduz e as estruturas tecnológicas nacionais podem reverter para padrões mais especializados. Portanto, a sucessão de paradigmas tecnológicos pode conduzir também a padrões de diversificação tecnológicos em "formato de U". Desta maneira, cada novo ciclo de desenvolvimento puxado pela exploração de novas oportunidades tecnológicas se corresponderia com estágios de diversificação seguidos de estágios de especialização tecnológica. A experiência histórica sugere que alguns países adquiriram fortes especializações aproveitando as oportunidades oferecidas por novos paradigmas, como por exemplo, Alemanha nas tecnologias Químicas, Farmacêuticas e Elétricas no período entre guerras; Japão em Transporte e Maquinaria no período pós-SGM; e Japão e Estados Unidos em Semicondutores e Eletrônica no período 1965-1990 (Vertova, 2001). O autor destaca ainda que desenvolver este tipo de especialização só é possível se os países detêm sistemas nacionais de inovação e ambientes institucionais apropriados.

Um segundo fator se refere aos efeitos das Grandes Corporações Transnacionais (GCT) sobre a especialização tecnológica dos países hospedeiros. Quando as GCT internacionalizam sua P\&D na busca e captura de competências tecnológicas (host-coutry-advantages), a especialização da internacionalização da GCT se corresponde com a apresentada pelos países hospedeiros (Patel e Vega, 1999; Le Bas e Sierra, 2002; Urraca-Ruiz, 2008; Miranda, 2014). Nestes casos, a ação das GCT reforça o perfil de especialização nacional, levando a estruturas tecnológicas mais concentradas (especializadas). Por outro lado, quando as GCT adotam estratégias de exploração de vantagens desenvolvidas no seu país de origem (home-country-advantages), elas poderão acompanhar ou não o padrão de especialização do país hospedeiro. O exercício de atividade inovadora em tecnologias onde o país hospedeiro não é especializado contribui para a expansão de sua base tecnológica tornando a estrutura tecnológica nacional mais diversificada. Este efeito pode acontecer tanto se as GCT se encontram instaladas no país hospedeiro quanto se não, dado que a captura de competências tecnológicas locais pode acontecer apenas mediante a inserção de inventores locais em equipes de pesquisa que trabalham para a GCT em qualquer lugar do mundo. As formas de inserção das 
GCT são muito variadas e sua ação será benéfica para o país hospedeiro sempre que os spillovers gerados possam ser internalizados mediante políticas específicas de transferência de conhecimento (Urraca-Ruiz, 2008; Wang, Xue e Liang, 2012; Kenney, Massini e Murtha, 2009; Gilman, 2010; Thomson, 2013).

Em terceiro lugar, o grau de especialização das estruturas tecnológicas nacionais também depende do tamanho do país (Archibugi e Pianta (1992) apud Cantwell e Vertova, 2004). Países menores tendem a ser mais especializados, bem em atividades vinculadas com suas vantagens absolutas internas quando não estão integrados com o mundo, ou bem em atividades que exploram vantagens comparativas no exterior quando estão integrados com o mundo (Holanda, Bélgica, Finlândia, etc.). Já países grandes, com uma demanda interna elevada, podem desenvolver estruturas tecnológicas mais diversificadas (Estados Unidos, Rússia, Brasil, China, Índia, etc.). Dessa forma, a evolução do padrão de especialização tende a ser mais suave em países grandes do que nos países pequenos os quais, em principio, não atingiriam os níveis de diversificação alcançados pelos grandes (Figura 1a). No entanto, a hipótese de especialização para país pequeno pode ser quebrada em presença de integração econômica, dado que o limite de escala imposto para se produzir de forma eficiente num número restrito de indústrias pode ser superado pelo comércio (exportações) e pelo investimento estrangeiro direto.

Figura 1. Diferenças nos estágios de diversificação tecnológica entre.

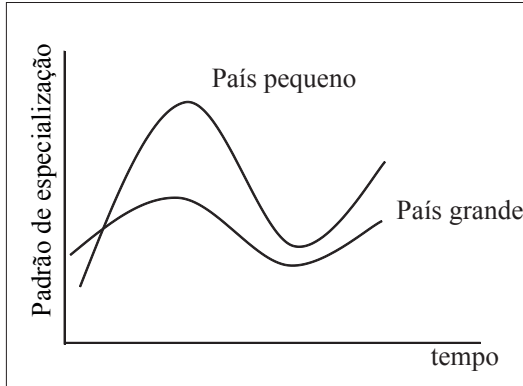

(a) Países grandes e pequenos

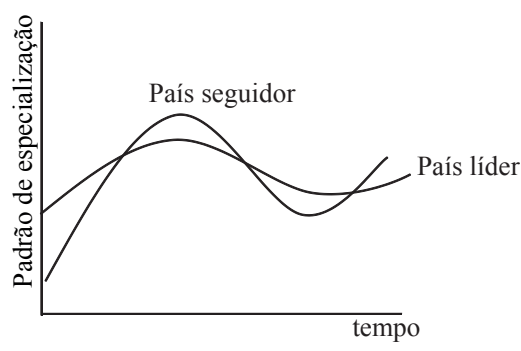

(b) Países líderes e seguidores

Fonte: Elaboração própria

Em quarto lugar, um fator que influencia o padrão de especialização tecnológico e sua persistência se refere à estabilidade e força de seus líderes tecnológicos (Malerba e Montobbio, 2003). O aproveitamento das oportunidades tecnológicas por parte de empresas pode transformá-las em líderes tecnológicos que acumulam 
e exploram vantagens de first mover durante longos períodos de tempo. Quando isto acontece, os países concentram em seus líderes tecnológicos as vantagens tecnológicas nacionais e elas podem persistir durante longos períodos de tempo. São os casos de BASF, Bayer e Hoechst em tecnologias Químico-Farmacêuticas ou Siemens, AEG e Telefunken em tecnologias Elétricas, na Alemanha; ou Toyota, Nissan, Isuzu, Honda, Mazda e Suzuki em Transporte e Mitsubishi Heavy Industry em Máquinas e equipamentos elétricos e eletrônicos no Japão (Vertova, 2001).

Finalmente, a evolução das estruturas tecnológicas pode seguir diferentes padrões em função da distância tecnológica em que se encontra o país com respeito à fronteira ao longo do processo de desenvolvimento. Países que se encontram próximos da fronteira tecnológica tenderão a apresentar maior estabilidade de suas estruturas, dado que o processo de acumulação de competências deverá se focar nos campos próximos da fronteira tecnológica em que o país já está especializado. Alternativamente, países mais distantes da fronteira que se encontram em processo de construção de novas competências mediante o catching-up, podem abandonar velhas especializações e construir outras novas, o que significa maior mobilidade e instabilidade das suas estruturas tecnológicas (Urraca-Ruiz, 2013a) (Figura 1b).

Desde os anos oitenta, a economia mundial passou a ser cada vez mais integrada, o que desencadeou diferentes processos de mudança estrutural nos países que fizeram parte desse processo com maior intensidade. Os países em desenvolvimento também passaram por diversos processos de integração, registrando fortes mudanças em suas estruturas tecnológicas com elevados graus de mobilidade entre campos técnicos (Archibugi e Pianta, 1994; Mancusi, 2001; Uchida e Cook, 2005; Urraca-Ruiz, 2013a; 2013b). A literatura identifica dois padrões diferentes de transformação estrutural em Ásia e em América Latina (Cimoli e Katz, 2003; Valli e Saccone, 2009; Hsieh, 2011). Por um lado, os países asiáticos conseguiram desenvolver especializações em tecnologias associadas ao paradigma de telecomunicações e audiovisual, particularmente no caso de China e Coreia do Sul. Já os países latino-americanos iniciaram seus processos de integração uma década depois e desenvolveram padrões de especialização em tecnologias vinculadas à exploração de recursos naturais (Urraca-Ruiz, 2013b). De acordo com diversos autores, o seguimento de diferentes padrões de especialização foi devido ao diferente ambiente institucional e às diferentes ações de políticas públicas estabelecidas em Ásia e em América Latina (Alcorta e Peres, 1998; Miozzo, 2002). Assim, enquanto em alguns países foi observada a redução da concentração absoluta da base técnica, em outros foi observado um período de crescimento da tendência à especialização (China e Coreia do Sul). 
Tomando como base essas observações, este trabalho tem dois objetivos: i) identificar mudanças no padrão de diversificação/especialização para um grupo de países asiáticos e latino-americanos desde o surgimento dos novos paradigmas tecnológicos nos anos 80; e ii) obter evidência empírica sobre a existência de estágios de diversificação na relação entre o padrão de diversificação tecnológica e o desenvolvimento dos países.

\section{BASE DE DADOS}

As estruturas tecnológicas praticamente só podem ser estimadas a partir da distribuição de patentes por campos tecnológicos. Isto acontece porque as estatísticas de patentes constituem a única fonte de informação que distribui o esforço formal e informal em inovação por campos de conhecimentos associados a tecnologias incorporadas em 'artefatos'. Ademais, as patentes oferecerem informações detalhadas para um grande número de países e para longos períodos de tempo, provendo uma base sólida para realizar comparações internacionais (Archibugi e Pianta, 1991; Mancusi, 2001).

No entanto, a distribuição nacional de patentes num momento específico de tempo revela uma distribuição de competências tecnológicas, mas não de capacitações no sentido de Archibugi e Coco (2005), dado que as patentes não conseguem medir o grau de complexidade do conhecimento envolvido quando são $\operatorname{produzidas}^{1}$, nem a contribuição da base científica à base técnica (Brusoni e Geuna, 2003). Adicionalmente, nem todos os esforços de mudança tecnológica resultam em patentes, de forma que algumas competências tecnológicas nacionais podem estar sendo subestimadas ${ }^{2}$.

Desde o ponto de vista da distribuição, as estatísticas de patentes estão sujeitas a viés setorial, dado que sua eficácia como forma de apropriação varia entre indústrias e entre tecnologias. Como consequência, uma distribuição homogênea de recursos à inovação não se refletirá numa distribuição homogênea

1 As patentes tratam apenas do conhecimento codificado e que não está incorporado (disembodied), deixando de abranger a complexidade do conhecimento que envolve as capacitações de um país. As capacitações envolvem indicadores de conhecimento incorporado e não incorporado, conhecimento tácito e codificado e geração e difusão de conhecimento. Mesmo existindo complementaridades entre essas categorias, o uso de patentes como fonte exclusiva de informação subestima o conjunto de aspectos que transforma competências em capacitações (Urraca-Ruiz, 2013).

2 As competências tecnológicas nacionais podem não resultar em patentes por não se basearem em tecnologias patenteáveis ou por decisões estratégicas das firmas. 
de patentes entre setores e tecnologias, concentrando-se mais em setores onde a propensão a patentear é maior. Por exemplo, o desenvolvimento de "produtos complexos" com múltiplas tecnologias (instrumentos ou certos equipamentos elétricos ou eletrônicos) pode dar origem a um conjunto de patentes complementares cuja divulgação pode facilitar a imitação por parte de firmas competidoras. Enquanto isso, nas chamadas "tecnologias discretas", um único produto pode estar protegido por um número reduzido de patentes (por exemplo, em tecnologias de alimentos e bebidas, têxteis, químico e farmacêutico). Apesar desta limitação, as diferentes propensões setoriais a patentear deverão ser relativamente homogêneas entre países.

Para este trabalho foi escolhida a base de dados de patentes do Escritório Europeu de Patentes (EPO), aplicável a 15 países europeus, o que elimina o viés nacional dos escritórios domésticos. As patentes foram agregadas a 29 campos técnicos de acordo com a classificação FISIR ${ }^{3}$, onde os campos tecnológicos relativos aos paradigmas tecnológicos dos oitenta são: Equipamentos Eletrônicos e Engenharia Elétrica, Telecomunicação e Audiovisual, Tecnologias da Informação, Semicondutores e Biotecnologia (NU. CEPAL, 2008, p.143). Esses novos paradigmas induziram o crescimento de setores produtivos como Computadores, Bens de Capital Eletrônicos, Softwares, Equipamentos de Telecomunicação, Computação e Audiovisual, Serviços de Informação (Freeman e Perez, 1988, p.53), Farmacêuticos e Biotecnologia e Semicondutores (Vertova, 2001, p.608).

As patentes foram atribuídas a cada país a partir da nacionalidade do inventor. Os países latino-americanos selecionados foram Argentina, Brasil e México. Juntos, estes países apresentaram crescimento da sua cota de patentes de $208,1 \%$ entre $1986-1990$ e $1996-2000$ e $159 \%$ entre $1996-2000$ e $2006-2010$. O grupo de países asiáticos formado por China, Coreia do Sul, Hong Kong, Índia, Cingapura e Taiwan reunia uma quantidade pequena de patentes no início dos anos 1980 , representando apenas $0,2 \%$ das patentes mundiais. O crescimento da cota de patentes destes países se acelerou nos 90 e na segunda metade dos anos 2000 passaram a uma cota de 7,3\% das patentes mundiais, registrando um crescimento de 333,6\% entre 1986-1990 e 1996-2000 e 484,7\% entre 1996-2000 e 2006-2010. Sem embargo, o crescimento entre os países foi bastante diferente. No primeiro período, Coreia do Sul e Cingapura foram os principais responsáveis por esse crescimento, enquanto no segundo China, Coreia do Sul, Índia e Taiwan apresentaram as maiores taxas.

3 Vide Anexo. Os campos 2 (Telecomunicações) e 3 (Audiovisual) foram agregados para melhorar a qualidade dos dados. 


\section{EVOLUÇÃO DOS PADRÕES DE DIVERSIFICAÇÃO/ESPECIALIZAÇÃO EM ÁSIA E AMÉRICA LATINA.}

A evolução da estrutura tecnológica foi observada mediante indicadores de concentração absoluta (Herfindahl-Hirschmann); de concentração relativa (ChiQuadrado) e do índice de especialização (Vantagem Tecnológica Revelada). O indice de Herfindahl-Hirschmann (normalizado, $H N_{j}$ ), representa uma medida da desigualdade na distribuição nacional de esforços entre atividades tecnológicas. Denominando $p_{i j}$ a distribuição percentual de patentes dos $i=1, \ldots, n$ campos técnicos do país $j$; o $H N_{j}$ se define como:

$$
H N_{j}=\frac{\sum_{i=1}^{n} p_{i j}{ }^{2}-1 / n}{1-1 / n} * 100
$$

O $H N_{j}$ varia entre 0 e 100 , sendo que quanto mais próximo a 100 , maior a desigualdade da distribuição, ou seja, maior será a concentração de esforços tecnológicos entre um menor número de campos técnicos.

A concentração relativa da estrutura tecnológica pode ser mensurada através do Chi-quadrado $\left(\chi^{2}\right)$. Este índice mede a distância relativa das distribuições percentuais entre campos de atividade tecnológica de um país (j) com respeito a uma área de referência (w), a qual é utilizada como benchmark da distribuição mais homogênea, dadas as diferenças intersetoriais a patentear (Huang e Miozzo, 2004; Urraca-Ruiz, 2013a). Formalmente,

$$
\chi^{2}=\sum\left[\frac{\left(p_{i j}-p_{w j}\right)^{2}}{p_{w j}}\right]
$$

onde $p_{i j}$ segue a mesma notação anterior e $p_{i w}$ é a distribuição percentual de patentes para $n$ campos técnicos de uma área de referência. Se um país possui a mesma distribuição percentual da área de referência, o valor do coeficiente $\chi^{2}$ será zero, o que significa que o país apresenta diversificação total. Caso contrário, quanto maiores as diferenças de distribuição das atividades tecnológicas em relação ao grupo de referência, maior será o valor da $\chi^{2}$. A distribuição do grupo de referência representa um benchmark da distribuição mais homogênea (diversificada) possível das diferenças intersetoriais da propensão a patentear entre campos técnicos. Neste trabalho, o grupo de referência foi composto pelos países membros da OCDE até 1964, exceto Turquia ${ }^{4}$.

4 Países admitidos na OCDE até 1964: Áustria, Bélgica, Dinamarca, França, Grécia, Islândia, Irlanda, Itália, Luxemburgo, Noruega, Países Baixos, Portugal, Reino Unido, Suécia, Suíça, 
Em geral, os países da América Latina mantiveram um baixo grau de concentração absoluta ao longo do período (Tabela 1). O México apresenta o $H N_{j}$ mais elevado do grupo no período inicial, mas com uma tendência de redução que foi interrompida apenas em 2006-2010. Neste último período, o incremento no valor do índice em relação ao período anterior pode indicar uma reversão de tendência para Brasil e México. Já a Argentina apresenta estabilidade do indicador, com apenas uma forte queda da concentração de esforços no período 1991-1995.

Os países asiáticos exibiram tendências bastante distintas entre si. China e Coréia do Sul tenderam a aumentar a concentração ao longo do período. Cingapura também apresentou uma tendência de intensificação da concentração absoluta, porém parece haver registrado uma mudança de tendência já no último período. Índia, Hong Kong e Taiwan reduziram o grau de especialização ao longo do período sendo que Taiwan e Hong Kong reverteram a concentração também no último período.

O indicador de concentração relativa analisa a diversificação como uma aproximação da estrutura tecnológica dos países selecionados com a estrutura formada pela agregação dos países da OCDE. Neste caso, uma estrutura mais diversificada não significa necessariamente que a distribuição seja mais homogênea, mas sim, que ela acompanha as diferentes propensões a patentear dos campos técnicos e sua evolução ao longo do tempo. O indicador mostra que a maior parte dos países apresentou tendências de diversificação que acompanharam seus processos de desenvolvimento econômico e tecnológico desde a segunda metade dos oitenta. Destacam-se as elevadas distâncias iniciais de Índia, Hong Kong e México, as quais foram se reduzindo ao longo do tempo. Alternativamente, China e Coreia do Sul seguiram tendências de concentração relativa (aumento de distâncias) a partir dos anos 2000. México e Brasil apresentaram redução da concentração relativa até os anos 2000 em que aumentaram suas distâncias com o mundo. Argentina reduziu sua concentração relativa ao longo de todo o período. Em Cingapura e Hong Kong houve aumento da concentração relativa da estrutura tecnológica na primeira metade dos anos 1990 seguida por uma tendência de redução que se estendeu até o último período. De forma geral, não parece se confirmar que os graus de concentração absoluta e relativa de esforços de inovação nacional estejam associados ao tamanho do país.

Um terceiro indicador é a Vantagem Tecnológica Revelada Normalizada (VTRN) definida como:

Turquia, Alemanha, Espanha, Canadá, USA e Japão. 


$$
\operatorname{VTRN}_{i j}=\operatorname{VTR} R_{i j}-1 / V T R_{i j}+1,
$$

onde

$$
V T R_{i j}=\frac{p_{i j}}{p_{i w}}, p_{i j, \text { e }} p_{i w} \text { seguem a mesma notação anterior. A VTRN }
$$
compara por quociente a distribuição percentual de patentes por campo técnico de um país com respeito à de uma área de referência. A VTR revela vantagens tecnológicas relativas, dado que a especialização está associada a um melhor desempenho tecnológico de um país com relação a uma média internacional (Malerba e Montobbio, 2003). A VTRN varia entre -1 e 1; quanto mais próximos de 1 os valores da VTRN, maior o fortalecimento das vantagens relativas em decorrência de um maior esforço relativo em determinados campos de conhecimento. Alternativamente, valores negativos da VTRN (ou próximos de -1) revelam desvantagens tecnológicas decorrentes de um escasso ou nulo esforço relativo em inovação ${ }^{5}$.

Com caráter geral, se confirma o distinto aproveitamento dos paradigmas tecnológicos dos oitenta entre os grupos de países asiáticos e latino-americanos (Tabela 2). Os países da América Latina aproveitaram em escassa medida os novos paradigmas dos anos oitenta para desenvolver vantagens tecnológicas próprias. Os três países latino-americanos se especializaram em Biotecnologia, embora com diferentes trajetórias: Argentina só adquiriu especialização após o período 1996-2000; Brasil manteve suas vantagens ao longo de todo o período e México foi perdendo especialização apesar da sua elevada especialização inicial. Dos três países latino-americanos, apenas México desenvolveu vantagens tecnológicas em Equipamentos Eletrônicos e Engenharia Elétrica, as quais podem estar associadas em alguma medida às maquilas do setor Eletrônico estadunidense no México.

5 Por se tratar de uma medida relativa, elevadas especializações podem ser reveladas a partir de um número muito baixo de patentes. Assim, países com pouca propensão a patentear costumam apresentar valores da VTR muito elevados e distribuições da VTR com elevados desvios padrão (Huang e Miozzo, 2004). 
Tabela 1. Indicadores do grau de especialização absoluto e relativo de Ásia e América Latina, 1986-1990 a 2006-2010.

1986-1990

1991-1995

1996-2000 2001-2005 2006-2010

Índice de Herfindahl-Hirschmann normalizado

\begin{tabular}{|c|c|c|c|c|c|}
\hline \multicolumn{6}{|l|}{ Ásia } \\
\hline China & 2,32 & 2,54 & 2,51 & 8,56 & 18,05 \\
\hline Cingapura & 4,07 & 7,60 & 6,71 & 7,04 & 4,22 \\
\hline Coreia do Sul & 4,09 & 8,11 & 8,09 & 13,61 & 13,38 \\
\hline Índia & 18,86 & 9,20 & 10,43 & 10,35 & 7,31 \\
\hline Hong Kong & 12,27 & 11,15 & 8,32 & 8,03 & 8,50 \\
\hline Taiwan & 6,36 & 4,59 & 4,30 & 3,98 & 6,59 \\
\hline \multicolumn{6}{|c|}{ América Latina } \\
\hline Argentina & 4,36 & 2,90 & 4,89 & 4,36 & 3,90 \\
\hline Brasil & 2,43 & 2,04 & 1,84 & 1,44 & 1,81 \\
\hline México & 6,15 & 2,72 & 2,39 & 2,30 & 3,29 \\
\hline \multicolumn{6}{|c|}{ Índice Chi-quadrado } \\
\hline \multicolumn{6}{|l|}{ Ásia } \\
\hline China & 0,37 & 0,40 & 0,32 & 0,38 & 1,04 \\
\hline Cingapura & 0,70 & 0,83 & 0,79 & 0,56 & 0,29 \\
\hline Coreia do Sul & 0,40 & 0,59 & 0,37 & 0,77 & 0,90 \\
\hline Hong Kong & 1,75 & 2,10 & 1,29 & 1,63 & 1,57 \\
\hline Índia & 2,54 & 1,36 & 1,88 & 1,57 & 1,01 \\
\hline Taiwan & 1,34 & 0,78 & 0,71 & 0,42 & 0,46 \\
\hline \multicolumn{6}{|c|}{ América Latina } \\
\hline Argentina & 1,34 & 0,79 & 0,93 & 0,91 & 0,81 \\
\hline Brasil & 0,62 & 0,55 & 0,35 & 0,42 & 0,64 \\
\hline México & 2,15 & 0,85 & 0,62 & 0,84 & 0,97 \\
\hline
\end{tabular}

Fonte: Elaboração própria. EPO, Space Bulletin (1978-2010). 
Os países asiáticos apresentaram distintos padrões de especialização. A China perdeu especialização relativa em Biotecnologia, mas manteve suas vantagens em Equipamentos Eletrônicos e Engenharia Elétrica (embora em ritmo decrescente). Em compensação, a China pendulou para microparadigmas relacionados com as Tecnologias da Informação, Telecomunicações e Audiovisual, onde começou a registrar especializações desde a segunda metade dos 2000. Hong Kong aumentou suas vantagens tecnológicas em Equipamentos Eletrônicos e Engenharia Elétrica, chegando a perder sua especialização inicial em Telecomunicações e Audiovisual. A Índia manteve suas vantagens relativas em Biotecnologia ao longo de todo o período, embora com certa tendência decrescente, ao tempo que, desde meados dos noventa, começou a adquirir vantagens em Tecnologia da Informação. A Coréia do Sul realizou um importante aproveitamento das possibilidades tecnológicas oferecidas pelos novos paradigmas dos oitenta, mantendo sua especialização inicial em Equipamentos Eletrônicos e Engenharia Elétrica e aumentando-a em Telecomunicações e Audiovisual. O país conseguiu desenvolver vantagens tecnológicas em Semicondutores e em Tecnologias da Informação. Taiwan também apresentou um forte aproveitamento dos paradigmas desenvolvendo e mantendo vantagens em Equipamentos Eletrônicos e Engenharia Elétrica, Telecomunicações e Audiovisual, Semicondutores e em Tecnologias da Informação. Por fim, Cingapura aproveitou amplamente as possibilidades abertas em Biotecnologia, Telecomunicações e Audiovisual, Semicondutores e Tecnologias da Informação dados os valores positivos da VTRN em praticamente todas as tecnologias e períodos.

A VTRN mostrou que os países construíram vantagens tecnológicas em campos técnicos associados aos setores produtivos que tiveram maior crescimento relativo no mesmo período. Os países asiáticos produtores e exportadores de equipamentos eletrônicos, como Coréia do Sul, Taiwan, Hong Kong e China (Central Intelligence Agency, 2010), mantiveram ou desenvolveram vantagens em Equipamentos Eletrônicos e Engenharia Elétrica. O México, que teve crescimento das exportações de eletrônicos por meio da produção de suas maquilas, também desenvolveu certas vantagens comparativas nestas tecnologias desde a metade da década de 90. Coréia do Sul, China e Taiwan tiveram entre suas principais indústrias (em valor agregado anual) a de Equipamentos de Telecomunicação (ibidem), particularmente a partir dos anos 2000. Cingapura e Índia, que apresentaram vantagens tecnológicas em Biotecnologia, são também importantes produtores e exportadores de produtos químicos e farmacêuticos. Argentina e Brasil também apresentaram vantagens tecnológicas em Biotecnologia, as quais se encontram associadas às indústrias intensivas em recursos naturais. 
Tabela 2. Vantagens Tecnológicas Reveladas Normalizadas nos campos associados aos novos paradigmas em Ásia e América Latina, 1986-1990 a 2006-2010.

\section{$\begin{array}{lllllllll}\text { AR } & \text { BR } & \text { MX } & \text { CN } & \text { SG } & \text { KR } & \text { HK } & \text { IN } & \text { TW }\end{array}$}

Biotecnologia

\begin{tabular}{llllllllll}
\hline $1986-1990$ & $-1,00$ & 0,15 & 0,78 & 0,14 & $-0,20$ & 0,24 & $-1,00$ & 0,63 & $-0,28$ \\
$1996-2000$ & $-0,02$ & 0,16 & 0,35 & 0,23 & 0,30 & 0,03 & $-0,59$ & 0,31 & $-0,28$ \\
$2006-2010$ & 0,46 & 0,10 & $-0,10$ & $-0,28$ & 0,35 & $-0,33$ & $-0,52$ & 0,11 & $-0,52$ \\
\hline
\end{tabular}

Engenharia Eletrônica e Equipamentos Eletrônicos

\begin{tabular}{llllllllll}
\hline $1986-1990$ & $-1,00$ & $-0,25$ & $-0,58$ & 0,07 & 0,44 & 0,31 & 0,66 & $-0,77$ & 0,21 \\
$1996-2000$ & $-0,29$ & $-0,04$ & 0,02 & 0,16 & 0,24 & 0,12 & 0,13 & $-0,69$ & 0,22 \\
$2006-2010$ & $-0,32$ & $-0,09$ & 0,15 & 0,08 & $-0,06$ & 0,26 & 0,38 & $-0,11$ & 0,35 \\
\hline
\end{tabular}

Telecomunicações e Audiovisual

\begin{tabular}{llllllllll}
\hline $1986-1990$ & 0,26 & $-0,19$ & $-0,22$ & $-0,24$ & $-0,14$ & 0,14 & 0,06 & $-1,00$ & $-0,21$ \\
$1996-2000$ & $-0,32$ & $-0,51$ & $-0,58$ & $-0,03$ & 0,29 & 0,42 & 0,24 & $-0,47$ & $-0,09$ \\
$2006-2010$ & $-0,58$ & $-0,55$ & $-0,61$ & 0,57 & 0,23 & 0,51 & $-0,27$ & $-0,02$ & 0,29 \\
\hline
\end{tabular}

Tecnologias da Informação

\begin{tabular}{llllllllll}
\hline $1986-1990$ & $-1,00$ & $-0,21$ & $-1,00$ & $-0,71$ & 0,50 & $-0,18$ & $-0,13$ & $-0,36$ & 0,01 \\
$1996-2000$ & $-0,35$ & $-0,80$ & $-0,71$ & $-0,29$ & 0,46 & 0,10 & 0,11 & 0,20 & 0,24 \\
$2006-2010$ & $-0,20$ & $-0,50$ & $-0,39$ & 0,04 & 0,18 & 0,20 & 0,02 & 0,15 & 0,29 \\
\hline
\end{tabular}

Semicondutores

\begin{tabular}{llllllllll}
\hline $1986-1990$ & $-1,00$ & $-0,20$ & $-1,00$ & $-1,00$ & 0,14 & $-0,11$ & $-1,00$ & $-1,00$ & $-0,38$ \\
$1996-2000$ & $-1,00$ & $-0,71$ & $-0,26$ & $-0,27$ & 0,60 & 0,00 & $-0,42$ & $-0,45$ & 0,45 \\
$2006-2010$ & $-0,30$ & $-0,93$ & $-1,00$ & $-0,21$ & 0,46 & 0,42 & $-0,20$ & $-0,61$ & 0,42 \\
\hline
\end{tabular}

Fonte: EPO, Space Bulletin (1978-2010). 


\section{ESTÁGIOS DE DIVERSIFICAÇÃO TECNOLÓGICA}

O trabalho empírico de Imbs e Wackziarg (2003) mostrou que ao longo do processo de desenvolvimento os países passam por estágios de diversificação da estrutura produtiva resultantes da influência do crescimento do nível de renda per capita e do grau de abertura comercial. Os países partem de uma estrutura especializada e baseada na dotação inicial de fatores, diversificam-se e crescem para que, a partir de certo nível de desenvolvimento, voltem a se especializar. Isto significa que não existe uma relação monotônica entre o crescimento de um país e a diversificação da sua estrutura produtiva (ibidem). A relação entre o padrão de diversificação produtivo e crescimento em países avançados e em países em processo de catching-up pode ser descrita por um "formato de U" (Carvalho e Kupfer, 2011). Todavia, esta curva não simétrica, pois o grau de diversificação da estrutura produtiva não retorna ao seu nível inicial. De maneira geral, a diversificação da estrutura produtiva é considerada importante nos estágios iniciais do desenvolvimento e deve ser estimulada para obter ritmos maiores de crescimento.

Apesar dos vínculos entre as estruturas produtiva e tecnológica, não é esperado que os estágios de diversificação produtiva e tecnológica configurem curvas idênticas tendo em vista que as possibilidades de conhecimento se expandem mais do que a capacidade de produção pela existência de spillovers tecnológicos entre campos técnicos. Portanto, o estágio diversificação pode se estender mais na base tecnológica do que na base produtiva.

Algumas evidências acerca da relação entre a diversificação tecnológica e o nível de desenvolvimento econômico (PIB per cápita) e tecnológico (estoque de patentes por trabalhador) se encontram nos gráficos das Figuras 1 e 2 . O índice de diversificação se define como:

$$
D_{j t}=\frac{1-\sum_{1}^{n} p_{j t}}{1-\frac{1}{n}}
$$

seguindo a mesma nomenglatura do índice Herfindahl-Hirschmann e foi calculado para três níveis de agregação dos campos técnicos: IPC-4 dígitos (633 campos técnicos); IPC-3 dígitos (120 campos técnicos) e IPC-2 dígitos (29 campos técnicos compatíveis com classificação FISIR em anexo). O estoque de patentes per capita $\left(S T K P_{j t}\right)$ foi calculado a partir dos fluxos acumulados de patentes ano a ano, sem considerar depreciação, sobre o emprego total (e) tal que

$$
S T K P_{j t}=\frac{\sum_{1}^{t} P}{e} .
$$


Figura 1. Diversificação tecnológica e nível de renda per capita (preços constantes - US\$ 2005/milhares de hab.)
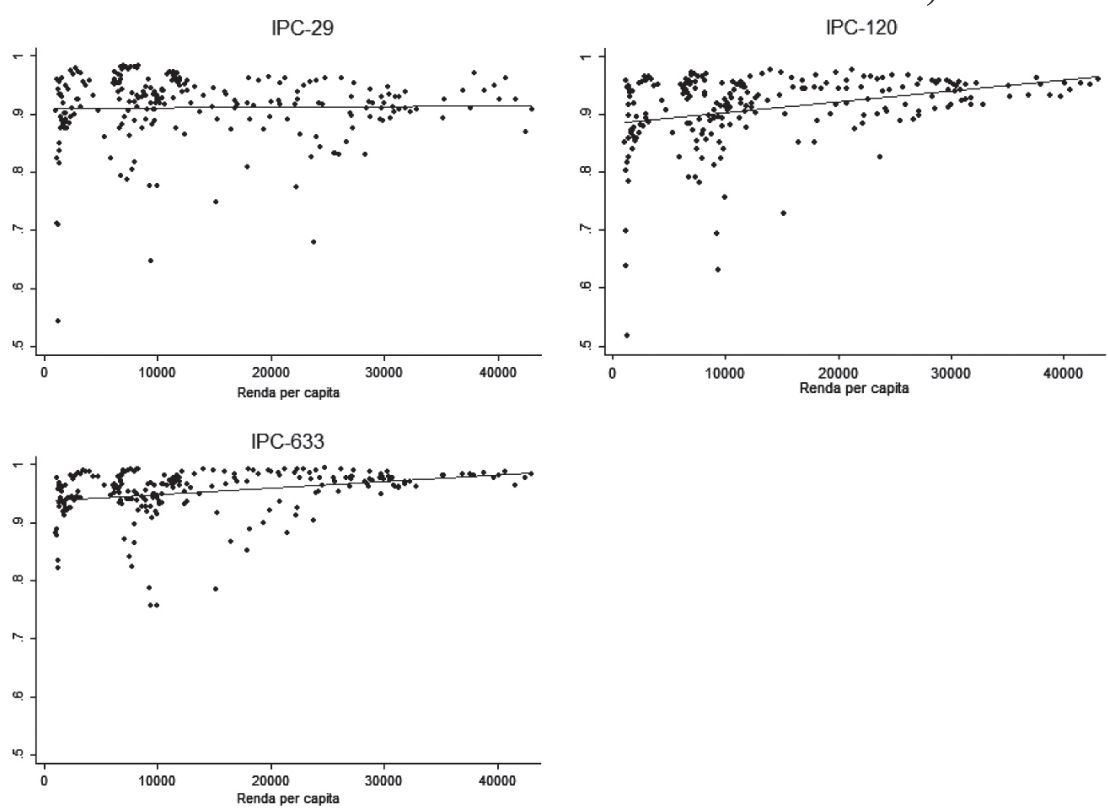

Fonte: Elaboração própria a partir de Space Bulletin, 1978-2011, EPO e Penn World Table 8.1.

$\mathrm{Na}$ classificação a dois dígitos (IPC-29) não é possível identificar uma relação entre diversificação e nível de desenvolvimento (Figura 1). Já para níveis maiores de desagregação, IPC-120 e IPC-633, observa-se uma leve inclinação positiva, o que pode ser considerado um indício de que após atingir um certo nível de desenvolvimento, o grau de diversificação tecnológica se torna mais estável. A relação entre o estoque de patentes per capita com o grau de diversificação mostra tendências similares (Figura 2). O ajuste dos valores é maior para a classificação mais desagregada (IPC-633). No entanto, a grande maioria dos pontos está localizada na área abaixo de 500 patentes per capita e não é possível observar mudança de tendência nessa relação. 
Figura 2. Diversificação tecnológica e estoque de patentes per capita (pessoas empregadas/milhares)

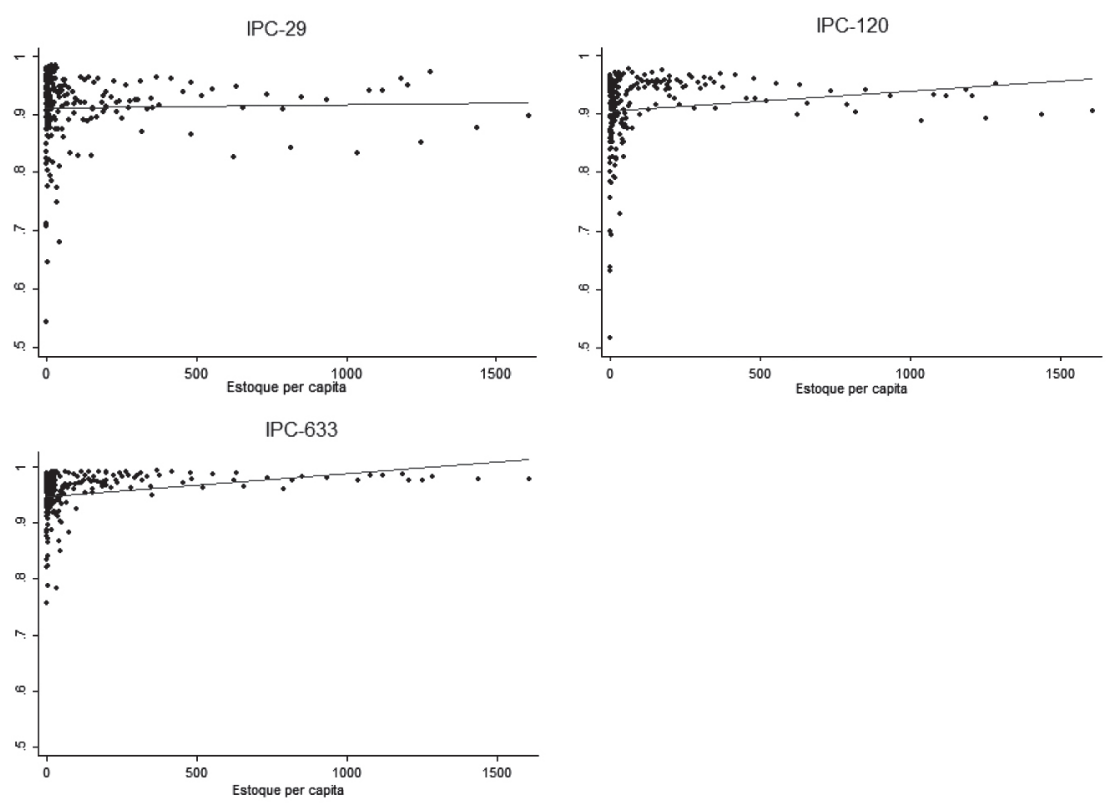

Fonte: Elaboração própria a partir de Space Bulletin, 1978-2011, EPO e Penn World Table 8.1.

Uma verificação mais apurada da relação entre o nível de desenvolvimento dos países e a diversificação tecnológica é a estimação da relação entre a variável de diversificação e as variáveis de desenvolvimento através de um modelo de painel. Os dados em painel combinam distintas características do comportamento de uma população ao longo do tempo e auxiliam na construção de modelos que comparam indivíduos com diferentes atributos. A estimação de um modelo de painel com efeitos fixos assume que características individuais de cada país (não correlacionadas com as características específicas dos demais países) podem viesar os resultados, razão pela qual a estimação elimina os efeitos que procedem dessas variáveis desconhecidas que não variam no tempo. A especificação da estimação dos modelos em painel de efeitos fixos são:

$$
\begin{array}{ll}
D_{j t}=\beta_{0}+\beta_{1} \ln G D P_{j t}+\alpha_{j} D U M_{j}+u_{j t}, & (\mathrm{EQ} 1) \\
D_{j t}=\beta_{0}+\beta_{1} \ln S T K P_{j t}+\alpha_{j} D U M_{j}+u_{j t}, & (\mathrm{EQ} 2)
\end{array}
$$


onde $\alpha_{j}(\mathrm{j}=1 \ldots 9)$ é o intercepto desconhecido para cada país; $D_{j t}$ é o grau de diversificação da estrutura tecnológica; $G D P_{j t}$ é a renda per capita; $S T K P_{j t}$ é o estoque de patentes por trabalhador; $D U M$ é uma variável dummy para cada pais e $u_{j t}$ é o termo de erro. A vantagem de se utilizar este intercepto para cada país é que se a variável não observada não varia no tempo, e por tanto, as mudanças no resultado são devidas a outras influências que não às decorrentes dos efeitos fixos (Stock e Watson, 2003).

As estimações foram realizadas com diferentes métodos para escolha dos critérios de efeito fixo ou efeito aleatório, sendo que os resultados de efeitos fixos foram estimados por Mínimos Quadrados Variável-Dummy (LSDV). Com a presença da dummy o valor de $\ln G D P_{j t}$ e de $\operatorname{lnSTKP_{jt}}$ é o efeito puro, pois a heterogeneidade dos países foi controlada. $\mathrm{O}$ modelo de efeitos aleatórios assume que a variação entre os países é aleatória e não correlacionada com as variáveis independentes incluídas no modelo. Neste caso, outras diferenças entre os países, que não o nível de renda per capita ou os estoques de patentes por trabalhador podem estar influenciando no comportamento da variável dependente. $\mathrm{O}$ teste de Hausmam rejeitou a hipótese nula de que os efeitos fixos e as variáveis explicativas não são correlacionados na EQ 1 (Hausman: 11,72 significativo ao 1\%), mas foi aceita na EQ 2 (Hausman: 0,76 sem significância). Por isso, o modelo de efeito fixo foi avaliado como o estimador mais eficiente no estudo das relações entre diversificação tecnológica e nível de desenvolvimento econômico, enquanto que o modelo com efeitos aleatórios se mostrou o mais consistente na relação entre diversificação tecnológica e desenvolvimento tecnológico.

No modelo de efeitos fixos o coeficiente da variação na renda per capita indica que o crescimento de $1 \%$ da renda per capita tem uma influência de 0,0431 sobre o grau de diversificação, em quanto que a cada $1 \%$ de variação do estoque de patentes corresponde a um efeito puro de 0,0143 sobre o índice de diversificação. Em todos os casos as variáveis mostraram relações positivas e significativas.

Para finalizar, o trabalho irá repetir o exercício sobre verificação de estágios de diversificação tecnológica ao longo do crescimento dos países utilizando a mesma técnica de estimação utilizada por Imbs e Wacziarg (2003). Trata-se da chamada técnica de LOWESS (Locally WEighted Scatterplot Smoothing). Este método utiliza regressão linear localmente ponderada para suavizar um gráfico do tipo $(x i, y i), i=1, \ldots, n$ facilitando a identificação de relações não lineares entre variáveis ao longo do tempo. O método consiste em montar um polinômio 
utilizando mínimos quadrados ponderados que ajusta cada ponto do conjunto de dados $(x i, y i)$ de acordo com pontos vizinhos à variável explicativa.

A função definida para atribuir pesos para os dados a cada intervalo é do tipo $W(x)=\left(1-|X|^{3}\right)^{3}$, em que X(i) representa a distância normalizada (ao longo do eixo $\mathrm{x}$ ) entre $x i$ e o valor sendo estimado. A normalização $\mathrm{X}$ é a distância máxima entre os pontos na regressão dentro de um intervalo definido previamente. O peso para $(x i, y i)$ é maior se $x i$ está perto do ponto estimado e pequeno caso contrário. $\mathrm{O}$ ajuste está completo após serem calculados valores a partir da função de regressão para cada um dos pontos do conjunto de dados. A curva resultante representa os valores ajustados.

Tabela 3. Estimações para os países no período 1985-2009, comparativo efeitos fixos e MQO

\begin{tabular}{|c|c|c|c|c|c|}
\hline \multicolumn{4}{|c|}{ Efeitos Fixos } & \multicolumn{2}{|c|}{ Efeitos Aleatórios } \\
\hline & EQ1 & EQ2 & & EQ1 & EQ2 \\
\hline$\beta_{0}$ & $0,5354 * * *$ & $0,8503 * * *$ & $\beta_{0}$ & $0,6906 * * *$ & $0,8602 * * *$ \\
\hline $\operatorname{lnGDP}$ & $0,0431 * * *$ & & $\operatorname{lnGDP}$ & $0,0285 * * *$ & \\
\hline $\operatorname{lnSTKP}$ & & $0,0143 * * *$ & $\operatorname{lnSTKP}$ & & $0,0145^{* * *}$ \\
\hline$\alpha \_$Brasil & $0,0562 * * *$ & $0,0289 * * *$ & & & \\
\hline a_China & $0,0870^{* * *}$ & $0,0190 * *$ & & & \\
\hline$\alpha \_$HongKong & $-0,0187$ & $0,0257 * * *$ & & & \\
\hline a_India & $0,0642 * * *$ & $(0,0209)^{* *}$ & & & \\
\hline$\alpha \_$Coreia & 0,0125 & 0,0089 & & & \\
\hline$\alpha \_$Mexico & $-0,0044$ & 0,002 & & & \\
\hline a_Cingapura & $(0,0332)^{* * *}$ & 0,0053 & & & \\
\hline a_Taiwan & $0,0251^{* *}$ & $0,0329 * * *$ & & & \\
\hline$N$ & 225 & 225 & $N$ & 225 & 225 \\
\hline$R^{2}$ & 0,384 & 0,495 & $\sigma u$ & 0,022 & 0,016 \\
\hline \multirow[t]{2}{*}{$R^{2}$ ajustado } & 0,358 & 0,474 & $\sigma e$ & 0,033 & 0,030 \\
\hline & & & $\rho$ & 0,304 & 0,231 \\
\hline$F(9,215)$ & 14,90 & 23,40 & Wald chi2(1) & 28,25 & 101,23 \\
\hline Prob $>F$ & 0,0000 & 0,0000 & Prob $>$ chi 2 & 0,000 & 0,000 \\
\hline
\end{tabular}

Significância: *, p $<0.05 ; * *, p<0.01 ; * * *, p<0.001$

Fonte: Elaboração própria. 
O método LOWESS foi aplicado com o objetivo de analisar a relação entre renda per capita e o grau de diversificação tecnológica (Índice de diversificação normalizado) entre 1984 e 2009. A análise prévia das mudanças no grau de especialização da estrutura tecnológica mostrou que a maioria dos nove países selecionados apresentou tendência a um baixo grau de especialização absoluta a partir da década de 1980. De acordo com as curvas formadas na Figura 1, a relação entre o grau de diversificação tecnológica e a renda per capita não é monotônica, mas após um crescimento da diversificação a estrutura permaneceu relativamente estável ao longo do período analisado.

Figura 1. Conjunto de curvas estimadas para os países - Índice de Diversificação Normalizado (IPC 4dgts) - Renda per capita - US\$ 2005/milhares hab. - (1984-2009).

\section{Lowess smoother}
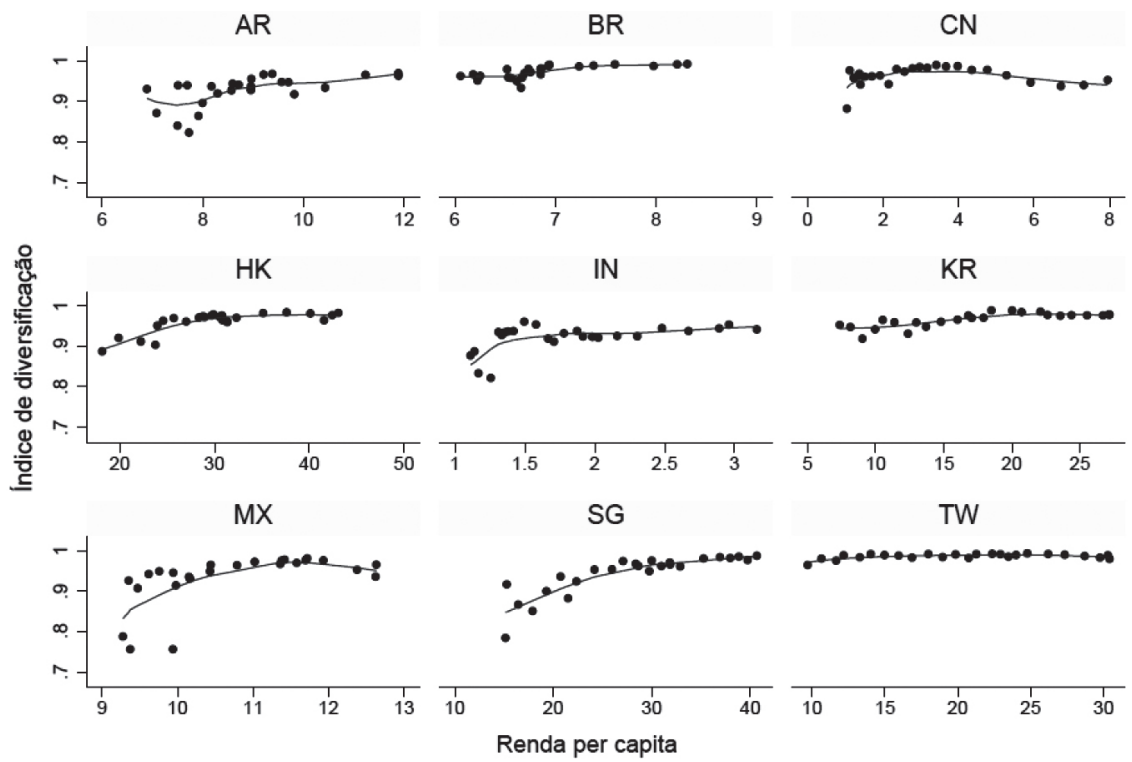

Fonte: Elaboração própria a partir de Space Bulletin, 1978-2011, EPO e Penn World Table 8.1.

Argentina, Índia, México e Cingapura foram os países que apresentaram um formato de curva com inclinação ascendente, ou seja, tiveram tendência à diversificação ao longo do período de catch-up tecnológico. As curvas de Taiwan, 
Brasil, China, Hong Kong e Coreia do Sul mostraram que os países passaram por um período longo de diversificação. Nenhum destes países apresentou formato semelhante ao esperado da sequência de estágios de especialização-diversificação-especialização relativa da estrutura tecnológica.

Em concordância com o observado na Figura 1, os países com curvas com formatos semelhantes, ou seja, que se encontram em estágios de diversificação semelhantes, possuem diferentes níveis de renda per capita, levando à conclusão de que os estágios estão associados a fatores outros além do ritmo de catching-up.

O aproveitamento dos paradigmas tecnológicos, observado através dos indicadores de vantagem tecnológica revelada, não parece ter influenciado uma mudança no grau de diversificação dos países analisados. Mesmo para Coreia do Sul e China, que tiveram rápido crescimento relativo da quantidade de patentes nos campos de Telecomunicações e Audiovisual, não se identifica mudança significativa no grau de diversificação, mas apenas pequenas flutuações.

\section{CONCLUSÕES}

Este trabalho tinha como objetivo procurar evidências de que a especialização em tecnologias associadas aos novos paradigmas levou à diversificação da estrutura tecnológica num grupo selecionado de países latino-americanos e asiáticos. Desde os anos 80 , estes países passaram por importantes mudanças em suas estruturas produtivas, de forma que os vínculos entre as estruturas produtiva e tecnológica podem ter contribuído para uma expansão da diversificação tecnológica. Além disso, a expansão das possibilidades de conhecimento e a existência de spillovers tecnológicos entre campos técnicos configuraram estruturas cada vez mais diversificadas. Essa tendência de diversificação da estrutura tecnológica, tanto absoluta quanto relativa, não parece estar associada ao tamanho das economias.

A concentração absoluta se reduziu ao longo do tempo em países com níveis iniciais elevados e manteve estável em países com níveis iniciais baixos. A concentração relativa se reduziu na maior parte dos países ao longo das três décadas, e ao mesmo tempo, foram se especializando em algumas das tecnológicas mais dinâmicas (relativas aos novos paradigmas tecnológicos dos 80). A especialização em tecnologias que oferecem elevadas oportunidades pode ter contribuído para a expansão da diversificação pelo fortalecimento da base tecnológica e pela existência de spillovers. 
A partir da análise dos dados em painel se observou que o grau de diversificação das estruturas tecnológicas está pouco associado aos níveis de renda per capita entre países. O nível acumulado de estoques de patentes tampouco se encontra associados ao grau de diversificação tecnológica. A associação entre variáveis não se da em nível, embora pode haver associação em termos dinâmicos que incorporem o papel da acumulação tecnológica e os caminhos passados de diversificação da estrutura tecnológica (path dependence).

O exame da relação entre diversificação e desenvolvimento pela técnica LOWESS revelou que tanto os países latino-americanos quanto os asiáticos passaram de uma especialização concentrada em poucos campos técnicos -característica dos estágios iniciais de desenvolvimento- para um estágio de diversificação duradouro. Não foram observadas diferenças significativas entre o formato de curva dos países que se encontram em níveis distintos de desenvolvimento.

Esforços adicionais para compreender os determinantes dos estágios ao longo do desenvolvimento dos países deverão ser realizados em futuros trabalhos. Tal é o caso da dimensão microeconômica da mudança tecnológica, isto é, qual o papel de liderança das empresas multinacionais no padrão de diversificação da estrutura tecnológica nacional. Por outro lado, o escopo do trabalho não permitiu realizar uma comparação direta entre a especialização produtiva e a especialização tecnológica. Assim, estudos que analisem em maior profundidade as relações entre estrutura produtiva e processo de construção de capacitações tecnológicas se fazem necessários. Estas conclusões devem permitir avançar no estudo da mudança tecnológica como dimensão da mudança estrutural. 
ANEXO - Correspondência entre a classificação IPC e os Campos Técnicos.

\begin{tabular}{|c|c|c|}
\hline & Campo Técnico & Código IPC \\
\hline 1 & $\begin{array}{l}\text { Componentes } \\
\text { elétricos }\end{array}$ & $\begin{array}{l}\text { F21H,F21K,F21L,F21M,F21P,F21Q,F21S,F21V,G05F,H01B,H01C,H01F, } \\
\text { H01G,H01H,H01J,H01K,H01M,H01R,H01T,H02B,H02G,H02H,H02J, } \\
\text { H02K,H02M,H02N,H02P,H05B,H05C,H05F,H05K }\end{array}$ \\
\hline 2 & $\begin{array}{l}\text { Audiovisual e } \\
\text { Telecomunicações }\end{array}$ & $\begin{array}{l}\text { G09F,G09G,G11B,H03F,H03G,H03J,H04N,H04R,H04S,H04W,G08C, } \\
\text { H01P,H01Q,H03B,H03C,H03D,H03H,H03K,H03L,H03M,H04B,H04H, } \\
\text { H04J,H04K,H04L,H04M,H04N,H04Q }\end{array}$ \\
\hline 4 & Informática & $\begin{array}{l}\text { G06C,G06D,G06E,G06F,G06G,G06J,G06K,G06M,G06N,G06Q,G06T, } \\
\text { G10L,G11C }\end{array}$ \\
\hline 5 & Semicondutores & H01L \\
\hline 6 & Ótica & G02B,G02C,G02F,G03B,G03C,G03D,G03F,G03G,G03H,H01S \\
\hline 7 & $\begin{array}{l}\text { Análises/Medidas/ } \\
\text { Controle }\end{array}$ & $\begin{array}{l}\text { G01B,G01C,G01D,G01F,G01G,G01H,G01J,G01K,G01L,G01M, } \\
\text { G01N,G01P,G01Q,G01R,G01S,G01V,G01W,G04B,G04C,G04D, } \\
\text { G04F,G04G,G05B,G05D,G07B,G07C,G07D,G07F,G07G,G08B,- } \\
\text { G08G,G09B,G09C,G09D,G12B }\end{array}$ \\
\hline 8 & Engenharia médica & A61B,A61C,A61D,A61F,A61G,A61H,A61J,A61L,A61M,A61N,A61P \\
\hline 9 & Química orgânica & $\mathrm{C} 07 \mathrm{C}, \mathrm{C} 07 \mathrm{D}, \mathrm{C} 07 \mathrm{~F}, \mathrm{C} 07 \mathrm{H}, \mathrm{C} 07 \mathrm{~J}, \mathrm{C} 07 \mathrm{~K}$ \\
\hline 10 & $\begin{array}{l}\text { Química } \\
\text { macromolecular }\end{array}$ & C08B,C08F,C08G,C08H,C08K,C08L,C09D,C09J,C09L,C13L \\
\hline 11 & $\begin{array}{l}\text { Fârmacos/ } \\
\text { Cosméticos }\end{array}$ & $\mathrm{A} 61 \mathrm{~K}$ \\
\hline 12 & Biotecnologia & $\mathrm{C} 07 \mathrm{G}, \mathrm{C} 12 \mathrm{M}, \mathrm{C} 12 \mathrm{~N}, \mathrm{C} 12 \mathrm{P}, \mathrm{C} 12 \mathrm{Q}, \mathrm{C} 12 \mathrm{R}, \mathrm{C} 12 \mathrm{~S}$ \\
\hline 13 & $\begin{array}{l}\text { Materiais/ } \\
\text { Metalurgia }\end{array}$ & $\begin{array}{l}\mathrm{B} 22 \mathrm{C}, \mathrm{B} 22 \mathrm{D}, \mathrm{B} 22 \mathrm{~F}, \mathrm{C} 01 \mathrm{~B}, \mathrm{C} 01 \mathrm{C}, \mathrm{C} 01 \mathrm{D}, \mathrm{C} 01 \mathrm{~F}, \mathrm{C} 01 \mathrm{G}, \mathrm{C} 03 \mathrm{C}, \mathrm{C} 04 \mathrm{~B}, \mathrm{C} 21 \mathrm{~B}, \\
\mathrm{C} 21 \mathrm{C}, \mathrm{C} 21 \mathrm{D}, \mathrm{C} 22 \mathrm{~B}, \mathrm{C} 22 \mathrm{C}, \mathrm{C} 22 \mathrm{~F}\end{array}$ \\
\hline 14 & $\begin{array}{l}\text { Produtos agrícolas } \\
\text { e alimentícios }\end{array}$ & $\begin{array}{l}\text { A01H,A21D,A23B,A23C,A23D,A23F,A23G,A23J,A23K,A23L,C12C, } \\
\text { C12F,C12G,C12H,C12J,C13D,C13F,C13J,C13K }\end{array}$ \\
\hline 15 & Processos técnicos & $\begin{array}{l}\text { A01N,A01P,C05B,C05C,C05D,C05F,C05G,C07B,C08C,C09B,C09C, } \\
\text { C09F,C09G,C09H,C09K,C10B,C10C,C10F,C10G,C10H,C10J,C10K, } \\
\text { C10L,C10M,C11B,C11C,C11D }\end{array}$ \\
\hline 16 & $\begin{array}{l}\text { Tratamentos de } \\
\text { superfície }\end{array}$ & $\begin{array}{l}\text { B01B,B01F,B01J,B01L,B02C,B03B,B03C,B03D,B04B,B04C,B05B,B } \\
\text { 06B,B07B,B07C,B08B,F25J,F26B }\end{array}$ \\
\hline 17 & $\begin{array}{l}\text { Processos de } \\
\text { materiais }\end{array}$ & $\begin{array}{l}\text { B05C,B05D,B32B,C23C,C23D,C23F,C23G,C25B,C25C,C25D,C25F, } \\
\text { C30B }\end{array}$ \\
\hline
\end{tabular}


18 Processos térmicos

19 Química de base

20 Meio-ambiente/ poluição

21 Máquinas e ferramentas

22 Motores/Bombas/ Turbinas

23 Componentes mecânicos

24 Manutenção/ Impressão

25 Aparelhos agrícolas e de alimentos

26 Transporte

27 Técnicas nucleares

28 Armamento/ Espacial

29 Bens de consumo

30 Engenharia civil
A41H,A43D,A46D,B28B,B28C,B28D,B29B,B29C,B29D,B31B,B31C, B31D,B31F,C03B,C08J,C14B,C14C,D01B,D01C,D01D,D01F,D01G,D 01H,D02G,D02H,D02J,D03C,D03D,D03J,D04B,D04C,D04G,D04H,D 05B,D05C,D06B,D06C,D06G,D06H,D06J,D06L,D06M,D06P,D06Q,D 21B,D21C,D21D,D21F,D21G,D21H,D21J

F22B,F22D,F22G,F23B,F23C,F23D,F23H,F23K,F23L,F23M,F23N,F23 Q,F24B,F24C,F24D,F24F,F24H,F24J,F25B,F25C,F27B,F27D,F28B,F2 $8 \mathrm{C}, \mathrm{F} 28 \mathrm{D}, \mathrm{F} 28 \mathrm{~F}, \mathrm{~F} 28 \mathrm{G}$

A62D,B01D,B09B,B09C,C02F,F01N,F23G,F23J

B21B,B21C,B21D,B21F,B21G,B21H,B21J,B21K,B21L,B23B,B23C, B23D,B23F,B23G,B23H,B23K,B23P,B23Q,B24B,B24C,B24D,B26D, B26F,B27B,B27C,B27D,B27F,B27G,B27H,B27J,B27K,B27L,B27M, $\mathrm{B} 27 \mathrm{~N}, \mathrm{~B} 30 \mathrm{~B}, \mathrm{~B} 81 \mathrm{~B}, \mathrm{~B} 81 \mathrm{C}, \mathrm{B} 82 \mathrm{~B}, \mathrm{~B} 82 \mathrm{Y}$

F01B,F01C,F01D,F01K,F01L,F01M,F01P,F02B,F02C,F02D,F02F,F02 G,F02K,F02M,F02N,F02P,F03B,F03C,F03D,F03G,F03H,F04B,F04C,F 04D,F04F,F23R

F15B,F15C,F15D,F16B,F16C,F16D,F16F,F16G,F16H,F16J,F16K,F16 L,F16M,F16N,F16P,F16S,F16T,F17B,F17C,F17D,G05G

B25J,B41B,B41C,B41D,B41F,B41G,B41J,B41K,B41L,B41M,B41N,B 65B,B65C,B65D,B65F,B65G,B65H,B66B,B66C,B66D,B66F,B67B,B6 7C,B67D

A01B,A01C,A01D,A01F,A01G,A01J,A01K,A01L,A01M,A21B,A21C, A22B,A22C,A23N,A23P,B02B,C12L,C13C,C13G,C13H

B60B,B60C,B60D,B60F,B60G,B60H,B60J,B60K,B60L,B60M,B60N, B60P,B60Q,B60R,B60S,B60T,B60V,B60W,B61B,B61C,B61D,B61F,B 61G,B61H,B61J,B61K,B61L,B62B,B62C,B62D,B62H,B62J,B62K,B6 2L,B62M,B63B,B63C,B63H,B63J,B64B,B64C,B64D,B64F

G01T,G21B,G21C,G21D,G21F,G21G,G21H,G21J,G21K,H05G,H05H

B63G,B64G,C06B,C06C,C06D,C06F,F41A,F41B,F41C,F41F,F41G,F 41H,F41J,F42B,F42C,F42D

A24B,A24C,A24D,A24F,A41B,A41C,A41D,A41F,A41G,A42B,A42C, $\mathrm{A} 43 \mathrm{~B}, \mathrm{~A} 43 \mathrm{C}, \mathrm{A} 44 \mathrm{~B}, \mathrm{~A} 44 \mathrm{C}, \mathrm{A} 45 \mathrm{~B}, \mathrm{~A} 45 \mathrm{C}, \mathrm{A} 45 \mathrm{D}, \mathrm{A} 45 \mathrm{~F}, \mathrm{~A} 46 \mathrm{~B}, \mathrm{~A} 47 \mathrm{~B}, \mathrm{~A} 47 \mathrm{C}$, $\mathrm{A} 47 \mathrm{D}, \mathrm{A} 47 \mathrm{~F}, \mathrm{~A} 47 \mathrm{G}, \mathrm{A} 47 \mathrm{H}, \mathrm{A} 47 \mathrm{~J}, \mathrm{~A} 47 \mathrm{~K}, \mathrm{~A} 47 \mathrm{~L}, \mathrm{~A} 61 \mathrm{Q}, \mathrm{A} 62 \mathrm{~B}, \mathrm{~A} 62 \mathrm{C}, \mathrm{A} 63 \mathrm{~B}$, A63C,A63D,A63F,A63G,A63H,A63J,A63K,B25B,B25C,B25D,B25F,B 25G,B25H,B26B,B42B,B42C,B42D,B42F,B43K,B43L,B43M,B44B,B4 4C,B44D,B44F,B68B,B68C,B68F,B68G,D04D,D06F,D06N,D07B,F25 D,G10B,G10C,G10D,G10F,G10G,G10H,G10K

E01B,E01C,E01D,E01F,E01H,E02B,E02C,E02D,E02F,E03B,E03C,E03 D,E03F,E04B,E04C,E04D,E04F,E04G,E04H,E05B,E05C,E05D,E05F,E 05G,E06B,E06C,E21B,E21C,E21D,E21F 


\section{REFERÊNCIAS BIBLIOGRÁFICAS}

Alcorta, L. \& Peres, W. (1998). Innovation Systems and Technological Specialisation in Latin America and the Caribbean. Research Policy, 26 (7-8), 857-881.

Archibugi, D. \& Coco, A. (2005). Measuring technological capabilities at the country level: A survey and a menu for choice. Research Policy, 34 (2), 175-194.

Archibugi, D. \& Pianta, M. (1991). Specialization and Size of Scientific Activities: A Bibliometric Analysis of Advanced Countries. Scientometrics, 22 (3), 341-358.

Archibugi, D. \& Pianta, M. (1994). Aggregate convergence and sectoral specialisation in innovation. Journal of Evolutionary Economics, 4 (1), 17-33.

Brusoni, S. \& Geuna, A. (2003). An international comparison of sectoral knowledge bases: persistence and integration in the pharmaceutical industry. Research Policy, 32 (10), 1897-1912.

Cantwell, J. \& Vertova, G. (2004). Historical evolution of technological diversification. Research Policy, 33 (3), 511-529.

Carvalho, L. \& Kupfer, D. (2011). Diversificação ou especialização: uma análise do processo de mudança estrutural da indústria brasileira. Revista de Economia Política, 31 (4), 618-637.

Central Intelligence Agency (2010). The World Factbook. Recuperado de https:// www.cia.gov/library/publications/the-world-factbook/index.html

Cimoli, M. \& Katz, J. (2003). Structural reforms, technological gaps and economic development: a Latin American perspective. Industrial and Corporate Change, 12 (2), 387-411.

Freeman, C. \& Perez, C. (1988). Structural Crises of Adjustment, Business Cycles and Investment Behaviour. En Dosi, G. \& Freeman, C. \& Nelson, R. \& Silverberg, G. \& Soete, L. (Eds.) Technical Change and Economic Theory. London: Francis Pinter.

Gilman, D. (2010). The New geography of global innovation. New York: Global Markets Institute at Goldman Sachs.

Hsieh, M. (2011). Similar opportunities, different responses: Explaining the divergent patterns of development between Taiwan and South Korea. International Sociology, 26 (3), 364-391.

Huang, H. T. \& Miozzo, M. (2004). Patterns of technological specialisation in Latin-American and East Asian countries: an analysis of patents and trade flows. Economic Innovation and New Technologies, 13 (7), 615-653.

Imbs, J. \& Wacziarg, R. (2003) Stages of diversification. American Economic Review, 93 (1), 63-86. 
Kenney, M., Massini, S. \& Murtha, T. P (2009). Off-shoring administrative and technical work: New fields for understanding the global enterprise introduction. Journal of International Business Studies, 40 (6), 887-900.

Le Bas, C. \& Sierra, C. (2002). Location versus home country advantages in R\&D activities: some further results on multinationals locational strategies. Research Policy, 31 (4), 589-609.

Malerba, F. \& Montobbio, F. (2003). Exploring factors affecting international technological specialization: the role of knowledge flows and the structure of innovative activity. Journal of Evolutionary Economics, 13 (4), 411-434.

Mancusi, M. (2001). International technological specialization in industrial countries: patterns and dynamics. Weltwirtschaftliches Archiv, 137 (4), 593-621.

Miozzo, M. (2002). Sectoral Specialisation in East Asia and Latin America Compared. Brazilian Journal of Political Economy, 22 (4), 48-68.

Miranda, P. (2014). A Internacionalização das Atividades Tecnológicas e a Inserção dos Países em Desenvolvimento: uma análise baseada em dados de patentes (Tesis doctoral). Universidade Estadual de Campinas, São Paulo.

Nelson, R. \& Winter, S. (1982). An evolutionary theory of economic change. Cambridge: Harvard University Press.

NU. CEPAL (2008). Structural Change and Productivity Growth - 20 Years Later. Old problems, new opportunities. Santiago: ECLAC.

Patel, P. \& Vega, M. (1999). Patterns of internationalisation of corporate technology: location vs. home country advantages. Research Policy, 28 (2-3), 145-155.

Perez, C. (2010). Technological revolutions and techno-economic paradigms. Cambridge Journal of Economics, 34 (1), 185-202.

Stock, J. H. \& Watson, M. W. (2003). Forecasting Output and Inflation: The Role of Asset Prices. Journal of Economic Literature, 41 (3), 788-829.

Teece, D. J., Rumelt, R., Dosi, G. \& Winter, S. (1994). Understanding corporate coherence. Theory and evidence. Journal of Economic Behavior and Organization, 23 (1), 1-30.

Thomson, R. (2013). National scientific capacity and R\&D offshoring. Research Policy, 42 (2), 517-528.

Urraca-Ruiz, A. (2008). Especialización y convergencia tecnológica en América Latina: el papel de las Empresas Multinacionales. Desarrollo Económico, 48 (189), 119-139.

Urraca-Ruiz, A. (2013a). The 'technological' dimension of structural change under market integration. Structural Change and Economic Dynamics, 27, 1-18.

Urraca-Ruiz, A. (2013b). Especialización tecnológica, captura y formación de competencias bajo integración de mercados: comparación entre Asia y América Latina. Economia e Sociedade, 22 (3), 641-667. 
Valli, V. \& Saccone, D. (2009). Structural Change and Economic Development in China and India. The European Journal of Comparative Economics, 6 (1), 101-129.

Vertova, G. (2001). National technological specialisation and the highest technological opportunities historically. Technovation, 21 (9), 605-612.

Wang, J., Xue, A. \& Liang, Z. (2012). Multinational R\&D in China: From home-country-based to host-country-based. Innovation: Management, policy \& practice 14 (2), 192-202.

(C) 2015 por los autores; licencia otorgada a la Revista Estudios Económicos. Este artículo es de acceso abierto y distribuido bajo los términos y condiciones de una licencia Atribución-No Comercial 3.0 Unported (CC BY-NC 3.0) de Creative Commons. Para ver una copia de esta licencia, visite http://creativecommons.org/ licenses/by-nc/3.0/ 
